\title{
OPTIMIZATION OF SUPERSONIC AXISYMMETRIC NOZZLES WITH A CENTER BODY FOR AEROSPACE PROPULSION
}

\author{
D. M. Davidenko ${ }^{1}$, Y. Eude ${ }^{1}$, and F. Falempin ${ }^{2}$ \\ ${ }^{1}$ ICARE - Institut de Combustion \\ Aérothermique, Réactivité et Environnement, CNRS \\ Av. de la Recherche Scientifique 1C, Orléans 45071, France \\ ${ }^{2}$ MBDA France \\ Av. Réaumur 1, Le Plessis Robinson 92358, France
}

\begin{abstract}
This study is aimed at optimization of axisymmetric nozzles with a center body, which are suitable for thrust engines having an annular duct. To determine the flow conditions and nozzle dimensions, the Vinci rocket engine is chosen as a prototype. The nozzle contours are described by 2 nd and 3rd order analytical functions and specified by a set of geometrical parameters. A direct optimization method is used to design maximum thrust nozzle contours. During optimization, the flow of multispecies reactive gas is simulated by an Euler code. Several optimized contours have been obtained for the center body diameter ranging from 0.2 to $0.4 \mathrm{~m}$. For these contours, Navier-Stokes (NS) simulations have been performed to take into account viscous effects assuming adiabatic and cooled wall conditions. The paper presents an analysis of factors influencing the nozzle thrust.
\end{abstract}

\section{INTRODUCTION}

Particular concepts of aerospace engines, e. g., continuous detonation wave engine [1], have an annular combustion chamber and require a supersonic nozzle with a center body for compatibility. To provide the maximum thrust for given geometric limitations (length and diameter), the nozzle contour must be carefully optimized. The modern approach to the nozzle design and optimization and related problems is discussed in [2].

Since the early works of Rao [3, 4] on the optimization of bell and spike nozzle contours, a significant effort has been made to apply the nozzle optimization, based on the calculus of variations and the method of characteristics, to flows with viscous boundary layers [5] and chemically nonequilibrium flows [6]. How-

This is an Open Access article distributed under the terms of the Creative Commons Attribution-Noncommercial License 3.0, which permits unrestricted use, distribution, and reproduction in any noncommercial medium, provided the original work is properly cited. 
ever, the method of characteristics, being well adapted to the design of several standard aerodynamic shapes, is difficult to generalize for an arbitrary shape and its applicability is limited to hyperbolic problems.

Finite-volume methods based on shock capturing numerical schemes are more attractive as they allow solutions with shocks, viscous layers, and subsonic flow zones. At the same time, such methods have two important shortcomings. First, they require predefined wall profiles; hence, they must be coupled, in automated manner, with a suitable design tool. Second, the flow solution is relatively expensive; therefore, the flow solver efficiency is a crucial point.

A simple design approach, compatible with direct optimization methods, is based on an analytical description of the nozzle contour by 2 nd and 3rd order polynomials. Nozzle contour optimization can be done by adjusting some geometrical parameters, e.g., coordinates and tangent angles at the end points, which define the polynomial coefficients. Applicability of this method was demonstrated for a classical bell nozzle [7] and an aerospike nozzle [8].

According to a more complicated design approach, the nozzle contour is specified by a set of points whose coordinates must be optimized. In the case of inviscid flow, the variation of the pressure integral can be locally approximated as a quadratic function of the radii of contour points [9]. The optimum contour is found by successively solving local optimization problems using the Newton method. In the case of viscous flow, methods based on the contour interpolation with cubic splines have been proposed. One of these methods [10] represents the nozzle contour as a linear combination of several basis functions, and the combination coefficients are found by solving a quadratic programming problem with the nonnegativity constraints. Another one [11] searches for optimum coordinates of control points using global optimization algorithms such as the genetic algorithm and its combination with the quadratic programming. Applicability of these methods to classical bell nozzles has been demonstrated.

In the present work, the nozzle contours are described by 2nd and 3rd order polynomials and a direct optimization method is used in combination with a twodimensional (2D) Euler solver to design maximum thrust nozzle contours. For a set of optimized nozzle contours, full NS simulations have been performed to take into account the skin friction and heat exchange on the walls. The following sections describe the design approach and optimization results.

\section{PROBLEM FORMULATION AND NUMERICAL METHODS}

\subsection{Nozzle Geometry}

For an annular nozzle, each wall contour is considered as a circular arc followed by a 2nd or 3rd order parabolic curve as shown in Fig. 1. For the lower (sub- 


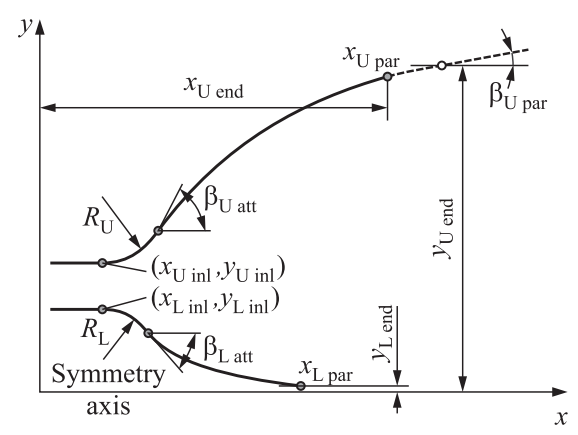

Figure 1 Schematic of the nozzle contour

script "L") and upper (subscript "U") contours, the following parameters are specified:

- starting point coordinates, $x_{\mathrm{inl}}$ and $y_{\mathrm{inl}}$;

- circular arc radius, $R$;

- contour angle at the attachment point, $\beta_{\text {att }}$;

- limiting coordinates, $x_{\mathrm{end}}$ and $y_{\mathrm{end}}$;

- parabolic contour abscissa, $x_{\text {par }}$, on the line $y=y_{\text {end }}$;

- tangent angle, $\beta_{\text {par }}$, at the point $\left(x_{\text {par }}, y_{\text {end }}\right)$.

This parameter set is sufficient to define a parabolic contour passing through the points $\left(x_{\mathrm{att}}, y_{\mathrm{att}}\right)$ and $\left(x_{\mathrm{par}}, y_{\mathrm{end}}\right)$ with the $\beta_{\mathrm{att}}$ tangent angle. The angle $\beta_{\text {par }}$ is used as a parameter if the upper contour is described by a cubic parabola. If $x_{\text {par }}<x_{\text {end }}$, the contour is limited by the radial coordinate, $y_{\text {end }}$, and the tangent angle at the end point, $\beta_{\mathrm{end}}$, is equal to $\beta_{\mathrm{par}}$. If $x_{\mathrm{par}}>x_{\mathrm{end}}$, the contour is limited by the axial coordinate, $x_{\text {end }}$, as shown in Fig. 1 , and $\beta_{\text {end }} \neq \beta_{\text {par }}$.

For the center body geometry, some particular cases considered below represent shapes with a cylindrical portion by setting $x_{\mathrm{L} \text { inl }}>x_{\mathrm{U} \text { inl }}$. The center body diameter, $D_{\mathrm{CB}}$, and the cylindrical portion length, $L_{\text {cyl }}$, are defined as follows: $D_{\mathrm{CB}}=2 y_{\mathrm{L} \text { inl }}, L_{\mathrm{cyl}}=x_{\mathrm{Linl}}-x_{\mathrm{U} \text { inl }}$.

\subsection{Nozzle Contour Optimization}

The nozzle contour optimization is a multidimensional problem. The chosen optimization algorithm is based on the direction set method [12] with simple restrictions on the optimization parameters. This means that fixed limits are 
specified for each parameter. The starting point is defined between the limits. To make an approach to the optimum, the $N$-dimensional optimization problem is treated as $N$ successive one-dimensional (1D) problems. A $1 \mathrm{D}$ optimization procedure searches for the optimum along each direction corresponding to a single parameter variation. The optimization is terminated when the distance between two successive approaches in the parameter space becomes less than the iteration accuracy. This algorithm is not very efficient in terms of the number of iterations but it is easy to implement. Besides, it is not applicable to the cases with multiple local optima. This shortcoming can be avoided by scanning the domain of possible solutions with large steps in order to localize the global optimum.

In the present realization, the optimization procedure operates on maximum 4 parameters: $\beta_{\mathrm{L} \text { att }}$ and $x_{\mathrm{L} \text { par }}$ for the lower contour, which is always quadratic; $\beta_{\mathrm{U} \text { att }}$ and $x_{\mathrm{U} \text { par }}$ for the quadratic upper contour or $\beta_{\mathrm{U} \text { att }}$ and $\beta_{\mathrm{U} \text { par }}$ for the cubic upper contour. For these parameters, the user specifies the limits, the starting point, and the iteration accuracy. An automatic optimization procedure sets the nozzle contour, for which the flow is simulated by the marching Euler code. The code evaluates the $x$-component of the integral pressure force along the lower and upper contours, which is used as the objective function to be maximized.

\subsection{Flow Simulation Methods}

A steady-state nozzle flow is simulated in 2D axisymmetric configuration using either the Euler or NS approach. Most of the Euler simulations, including all the optimization, have been made with a particular code that realizes a $2 \mathrm{nd}$ order accurate space-marching scheme. The space marching [13] is performed along the principal flow direction; an implicit Runge-Kutta integration scheme is applied to ensure a robust solution procedure for a chemically reacting flow. The computational mesh is automatically generated during each simulation. It has 50 points in the $y$-direction uniformly distributed and arranged along parallel vertical lines. The mesh step in the $x$-direction is controlled by a given Courant number. Numerical tests have shown that the integral pressure forces applied to the nozzle are virtually independent (to $0.01 \%$ ) of the Courant number variation within the range $0.5-2$ and are the same for a twice denser mesh in the $y$ direction.

Navier-Stokes simulations as well as some Euler simulations have been performed with the Fluent ${ }^{\circledR} 6.3$ commercial code. Implicit integration and a 2nd order accurate space approximation were chosen for the solution procedure. The structured mesh had 70 cells in the transversal direction and the cell number in the longitudinal direction was of the order of 500. The mesh was clustered near the walls in order to properly resolve the boundary layers. The minimum cell size was about $60-70 \mu \mathrm{m}$. 
A finite-rate kinetic model was adopted to describe the nonequilibrium chemistry during the expansion of combustion products. This model was represented by a kinetic mechanism including 6 species $\left(\mathrm{H}_{2}, \mathrm{O}_{2}, \mathrm{H}_{2} \mathrm{O}, \mathrm{H}, \mathrm{O}\right.$, and $\left.\mathrm{OH}\right)$ and 7 reversible chemical reactions [14]. For pure species, temperature-dependent thermodynamic properties were described by standard polynomials [15].

The molecular viscosity and conductivity of the gaseous mixture were evaluated according to the kinetic theory [16] and approximated as temperature functions under the following assumptions: the mixture was at chemical equilibrium whereas the temperature and pressure were isentropically related. Species diffusivities were considered with respect to fully recombined combustion products, containing only major species, and approximated as functions of the pressure and temperature.

As a turbulence model, the Wilcox $k-\omega$ model [17] was used together with the compressibility correction. The turbulent Prandtl and Schmidt numbers were taken equal to 0.85 .

\subsection{Engine Specifications}

In the present study, the optimization method was applied to an annular nozzle, for which the nozzle of the Vinci rocket engine was taken as a prototype. Based on previous Internet publications made by Astrium and Snecma, the following data were fixed for the study:

- nozzle exit diameter $2.15 \mathrm{~m}$;

- mass flow rate $33.7 \mathrm{~kg} / \mathrm{s}$;

- chamber pressure 6.08 $\mathrm{MPa}$; and

$-\mathrm{O}_{2} / \mathrm{H}_{2}$ mixture ratio 5.84 .

From available photo [18] of the engine, one could roughly estimate the longitudinal dimensions of the diverging nozzle:

- length of the fixed part $1.4 \mathrm{~m}$; and

- total deployed length $3.4 \mathrm{~m}$.

Ideal equilibrium simulations provided the following estimations for the throat diameter and mean flow conditions in the throat section:

- throat diameter $0.1323 \mathrm{~m}$;

- total pressure 5.781 MPa;

- static pressure 3.334 MPa; 
- total temperature $3529 \mathrm{~K}$;

- static temperature $3340 \mathrm{~K}$; and

- velocity $1548 \mathrm{~m} / \mathrm{s}$.

The corresponding ideal engine performance was as follows:

- engine specific impulse $4842 \mathrm{~m} / \mathrm{s}$ (4581 m/s (official data));

- engine vacuum thrust $163.2 \mathrm{kN}$ (155 kN (official data)); and

- supersonic nozzle thrust $65.2 \mathrm{kN}$.

\subsection{Input Data for Nozzle Contour Design}

Different diameters of the center body chosen for the optimization were $D_{\mathrm{CB}}=0$, $0.2,0.3$, and $0.4 \mathrm{~m}$. Note that $D_{\mathrm{CB}}$ defines the $y_{\mathrm{L} \text { inl }}$ coordinate whereas $y_{\mathrm{U} \text { inl }}$ is determined from the throat area, which is the same for all $D_{\mathrm{CB}}$. The axial position of the throat section is defined by the $x_{\mathrm{U} \text { inl }}$ coordinate that is set equal to 0 . For most of the cases considered below, the circular arc radii, $R_{\mathrm{U}}$ and $R_{\mathrm{L}}$, were taken equal to the throat diameter. For the center body with $L_{\text {cyl }}>0, R_{\mathrm{L}}$ was limited by the condition: $R_{\mathrm{L}} \geq L_{\text {cyl }} / 2$.

Respecting the Vinci nozzle dimensions, the upper contour was limited as follows: $x_{\mathrm{U} \text { end }}=3.4 \mathrm{~m}$ (total deployed length) and $y_{\mathrm{U} \text { end }}=1.075 \mathrm{~m}$ (half of the exit diameter). The limitations for the lower contour were $x_{\mathrm{L} \text { par }} \leq 1.4 \mathrm{~m}$ (length of the fixed part of the Vinci nozzle) and $y_{\mathrm{L} \text { end }} \approx 0$.

The tolerances respected during the contour optimization were the following: $0.01 \mathrm{~m}$ for $x_{\mathrm{L} \text { par }}$ and $x_{\mathrm{U} \text { par }} ; 0.1$ for $\beta_{\mathrm{L} \text { att }}$ and $\beta_{\mathrm{U} \text { att }}$; and 0.01 for $\beta_{\mathrm{U} \text { par }}$.

\subsection{Boundary Conditions}

For the nozzle flow simulation, a uniform sonic flow was imposed in the throat. This is a rough approximation because the sonic line is usually curved [19]; however, its exact shape depends on the duct configuration upstream from the nozzle throat that is not considered in the present study. The gas composition in the throat corresponded to the chemical equilibrium and was specified in terms of species mass fractions:

\begin{tabular}{cccccc}
\hline $\mathrm{H}_{2} \mathrm{O}$ & $\mathrm{H}_{2}$ & $\mathrm{O}_{2}$ & $\mathrm{OH}$ & $\mathrm{H}$ & $\mathrm{O}$ \\
\hline 0.89866 & 0.04008 & 0.00704 & 0.04789 & 0.00272 & 0.00361 \\
\hline
\end{tabular}


The mass fractions of $\mathrm{HO}_{2}$ and $\mathrm{H}_{2} \mathrm{O}_{2}$, which were not taken into account in the present study, were of the order of $10^{-5}$ or less and their thermodynamic effect was negligible. One can appreciate the dissociation level noting that the composition of fully recombined combustions products was 0.96138 of $\mathrm{H}_{2} \mathrm{O}$ and 0.03862 of $\mathrm{H}_{2}$.

For the NS simulations, a turbulence intensity of $5 \%$ and a turbulence length scale of $0.5 \mathrm{~mm}$ were imposed in the throat. No-slip conditions were used for the flow velocity on the walls. Two kinds of thermal wall conditions were considered: adiabatic and constant temperature of $1300 \mathrm{~K}$.

The outflow conditions were not important as the simulated flow velocity was supersonic everywhere in the exit cross section. The nozzle was supposed to operate in vacuum.

\section{RESULTS AND DISCUSSION}

\subsection{Validation of the Optimization Method}

The first test was related to the classical bell nozzle. A nonreacting perfect gas was considered with the following properties: ratio of specific heats 1.2 and gas constant $630 \mathrm{~J} /(\mathrm{kg} \cdot \mathrm{K})$. The aforementioned static pressure and temperature in the throat were imposed together with a Mach number of 1.01. The nozzle contour was designed using several methods: the method of characteristics [17], optimum quadratic contour, and optimum cubic contour. For the method of characteristics, the circular contour upstream from the attachment point was fixed whereas the downstream contour was designed along a streamline. The $\beta_{\mathrm{U} \text { att }}$ angle was adjusted to obtain a contour passing through the point $\left(x_{\mathrm{U} \text { end }}\right.$, $\left.y_{\mathrm{U} \text { end }}\right)$. It should be noted that the end point had the same coordinates in the other cases. The results are summarized in Table 1. For the two optimum contours, the thrust was obtained from Euler simulations.

One can note that the angles obtained for the quadratic contour are significantly different from the corresponding values provided by the method of char-

Table 1 Contour angles and normalized thrust for the classical nozzle

\begin{tabular}{lccc}
\hline \multicolumn{1}{c}{ Configuration } & $\beta_{\mathrm{U} \text { att }}$ & $\beta_{\mathrm{U}_{\mathrm{par}}}$ & $F_{N} / F_{N}^{*}{ }^{\dagger}$ \\
\hline Characteristics & $32.1^{\circ}$ & $8.0^{\circ *}$ & 0.9829 \\
Quadratic optimum & $40.3^{\circ}$ & $10.1^{\circ *}$ & 0.9816 \\
Cubic optimum & $36.1^{\circ}$ & $8.2^{\circ}$ & 0.9857 \\
\hline
\end{tabular}

*Nonoptimized angle.

${ }^{\dagger}$ Nozzle thrust normalized by the ideal nozzle thrust $63 \mathrm{kN}$ in the perfect gas case. 


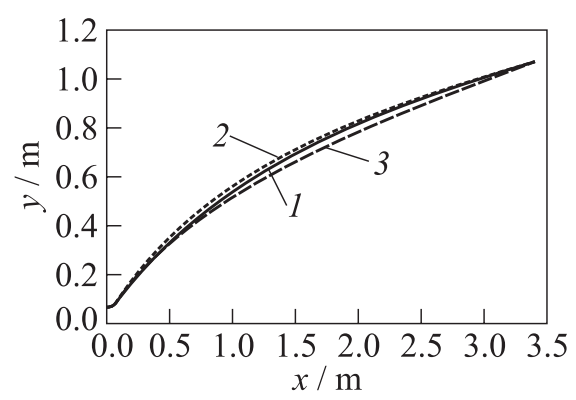

Figure 2 Comparison of nozzle contours obtained for the classical configuration: 1 characteristics; 2 - quadratic; and 3 - cubic

acteristics. The optimum cubic contour is in closer agreement with the method of characteristics and it gives higher thrust. The three contours are plotted in Fig. 2.

\subsection{Factors Influencing Thrust}

The engine thrust, $F_{E}$, can be considered as the flow momentum in the nozzle throat plus the nozzle thrust, $F_{N}$, which is represented by a sum of the integral pressure force, $F_{p}$, and the skin friction drag, $F_{\nu}$, acting in the $x$-direction. The most important factors that influence these integral forces are the contour shape, chemical reactions, and wall thermal conditions. The contribution of each factor could be assessed from the following considerations. Zero-dimensional (0D) equilibrium computations provided the highest theoretical level for the nozzle thrust $F_{N}^{*}=65.2 \mathrm{kN}$ and the engine thrust $F_{E}^{*}=163.17 \mathrm{kN}$. By progressively increasing the complexity of the numerical approach and choosing different options, it was possible to evaluate the influence of each factor more or less independently. The results obtained for the classical bell nozzle are given in Table 2. The numbers represent differences in the pressure force, $\Delta F_{p}=F_{p}-F_{N}^{*}$, nozzle thrust, $\Delta F_{N}=F_{N}-F_{N}^{*}$, and engine thrust, $\Delta F_{E}=F_{E}-F_{E}^{*}$, as well as the viscous force, $F_{\nu}$, with respect to the highest theoretical thrust.

The most important losses are obtained if the gas composition is frozen in the nozzle but this is not a real case. With the finite-rate chemistry, the nozzle thrust losses are within 1\%. Independently of the numerical approach and the nozzle shape, the mean mass fraction of $\mathrm{H}_{2} \mathrm{O}$ in the exit cross section is about 0.961 , i. e., close to the equilibrium. Due to the nozzle shape, the thrust losses increase by $1.3 \%-4.6 \%$. As compared to the conical nozzle, more than $3 \%$ of thrust can be gained if the nozzle contour is optimized. With the viscous effects, the flow is less expanded, thus, the pressure force increases with respect to the inviscid case. However, the net effect is negative because the skin friction drag represents 
Table 2 Influence of different factors on nozzle and engine thrust

\begin{tabular}{|c|c|c|c|c|c|c|c|}
\hline Approach & Chemistry & Form & Wall & $\begin{array}{c}\Delta F_{p} / F_{N}^{*} \\
\%\end{array}$ & $\begin{array}{c}F_{\nu} / F_{N}^{*} \\
\%\end{array}$ & $\begin{array}{c}\Delta F_{N} / F_{N}^{*} \\
\%\end{array}$ & $\begin{array}{c}\Delta F_{E} / F_{E}^{*}, \\
\%\end{array}$ \\
\hline $0 \mathrm{D}$ & Frozen & & & -12.1 & & -12.1 & -4.8 \\
\hline $1 \mathrm{D}$ & Finite rate & Conical & & -0.7 & & -0.7 & -0.3 \\
\hline $2 \mathrm{D}$ & & Conical & & -5.3 & & -5.3 & -2.1 \\
\hline Euler & & Cubic & & -2.0 & & -2.0 & -0.8 \\
\hline $2 \mathrm{D}$ & & & Adiabatic & 0.7 & -5.2 & -4.5 & -1.8 \\
\hline $\mathrm{NS}$ & Finte rate & Cunic & $T_{w}=1300 \mathrm{~K}$ & -0.7 & -5.8 & -6.5 & -2.6 \\
\hline
\end{tabular}

more than $5 \%$ of the nozzle thrust. Finally, the wall cooling is responsible for a 2 percent nozzle thrust loss due to the pressure force reduction and viscous force increase.

\subsection{Optimization of Nozzles with a Center Body for Inviscid Flow}

This section presents the results for a nonequilibrium inviscid flow in the nozzle. A parametric study has been conducted for $D_{\mathrm{CB}}=0.4 \mathrm{~m}$ and both types of the upper contour. In the case of nozzle with quadratic upper contour, all the four optimization parameters were varied. It is found that the maximum thrust is always obtained when $x_{\mathrm{U} \text { par }}=x_{\mathrm{U} \text { end }}$. The effect of the other parameters, $x_{\mathrm{L} \text { par }}$, $\beta_{\mathrm{L} a t t}$, and $\beta_{\mathrm{U} \text { att }}$, is illustrated in Fig. 3 for the normalized nozzle thrust, $F_{\mathrm{N}}$, and its components corresponding to the lower contour, $F_{\mathrm{L}}$, and the upper contour, $F_{\mathrm{U}}$. Component $F_{\mathrm{L}}$ is independent of $\beta_{\mathrm{U} \text { att }}$ within the considered domain. For a constant lower angle, $\beta_{\mathrm{L} \text { att }}$, the greatest $F_{\mathrm{L}}$ is found on the boundary corresponding to a straight line. The shortest straight contour is the best one to maximize $F_{\mathrm{L}}$. However, this is not the case for $F_{\mathrm{U}}$, for which the greatest value is found for the longest possible center body with a straight contour. As a matter of fact, considering the $x_{\mathrm{L} \text { par }}-\beta_{\mathrm{L} \text { att }}$ plane in Fig. $3 b$, one can note that maximum $F_{\mathrm{U}}$ is obtained at the crossing of two limitations: the curve representing straight lower contours, and the center body length limitation $x_{\mathrm{L} \text { par }}=1.4 \mathrm{~m}$. The nozzle total thrust, $F_{\mathrm{N}}$, exhibits a similar behavior. One can conclude from this analysis that the variation of the center body shape makes a stronger effect on $F_{\mathrm{U}}$ than on $F_{\mathrm{L}}$.

In the case of nozzle with cubic upper contour, both contour lengths were fixed: $x_{\mathrm{L} \text { par }}=1.4 \mathrm{~m}$ and $x_{\mathrm{U} \text { par }}=3.4 \mathrm{~m}$. The effect of the contour angles, $\beta_{\mathrm{L} \text { att }}$, $\beta_{\mathrm{U} \text { att }}$, and $\beta_{\mathrm{U} \text { par }}$, on $F_{\mathrm{L}}, F_{\mathrm{U}}$, and $F_{\mathrm{N}}$ is illustrated in Fig. 4. Once again, the variation of $\beta_{\mathrm{L} \text { att }}$ acts in opposite ways on $F_{\mathrm{L}}$ and $F_{\mathrm{U}}$. As in the previous case, the maximum nozzle thrust is obtained for the straight center body contour. One can see an optimum of $F_{\mathrm{N}}$ in the plane $\beta_{\mathrm{U} \text { att }}-\beta_{\mathrm{U} \text { par }}$ at $\beta_{\mathrm{L} \text { att }}=-7.78^{\circ}$. 


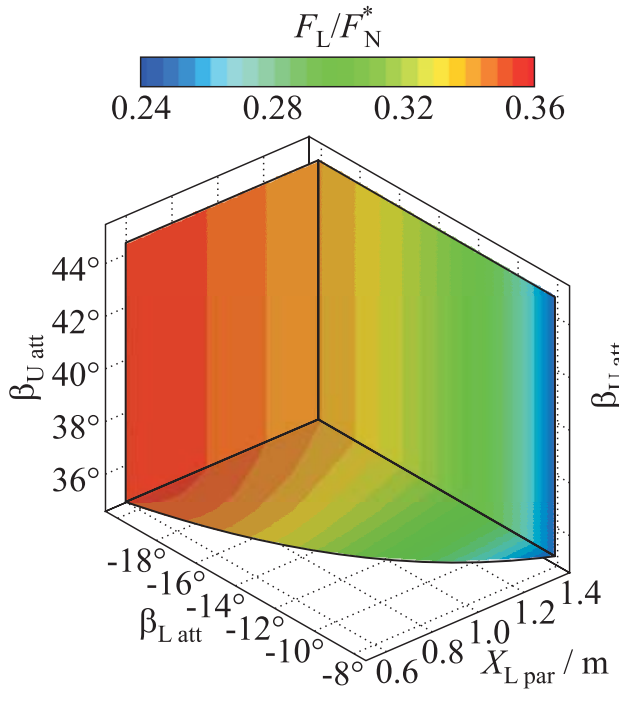

(a)

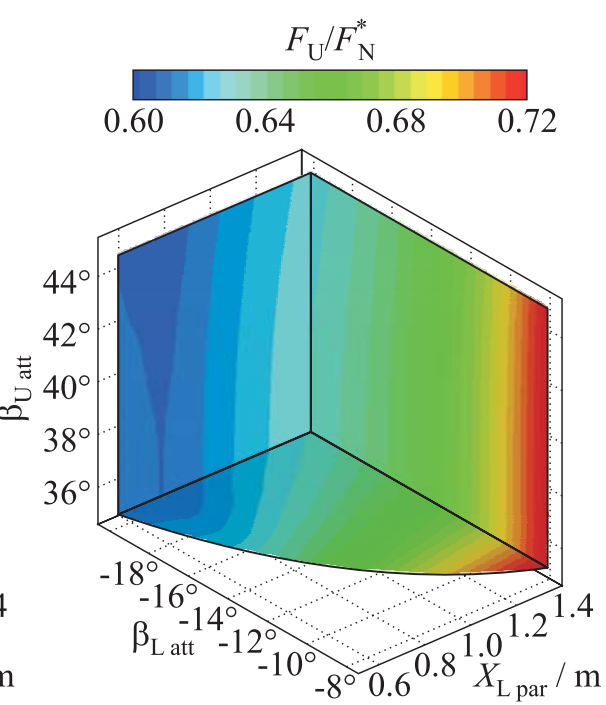

(b)

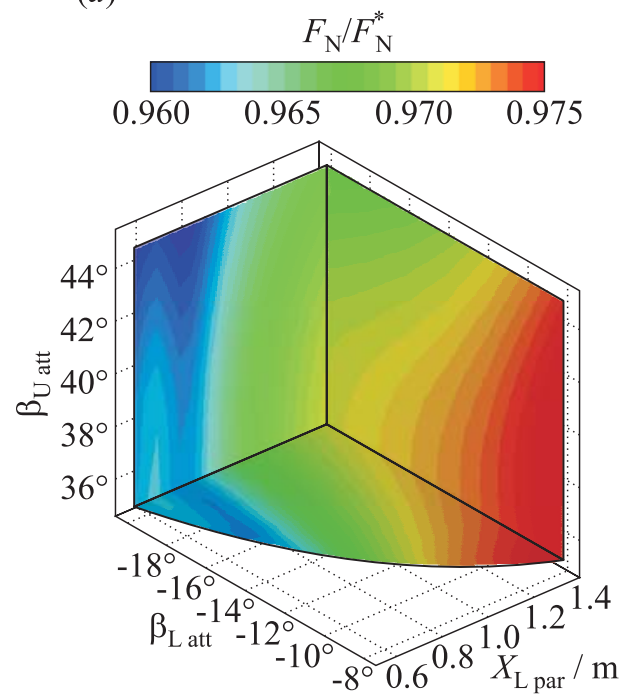

(c)

Figure 3 Normalized pressure forces for $D \mathrm{CB}=0.4 \mathrm{~m}$ and quadratic upper contour: lower contour $(a)$; upper contour (b); and their sum $(c)$. (Refer Davidenko et al., p. 684.) 


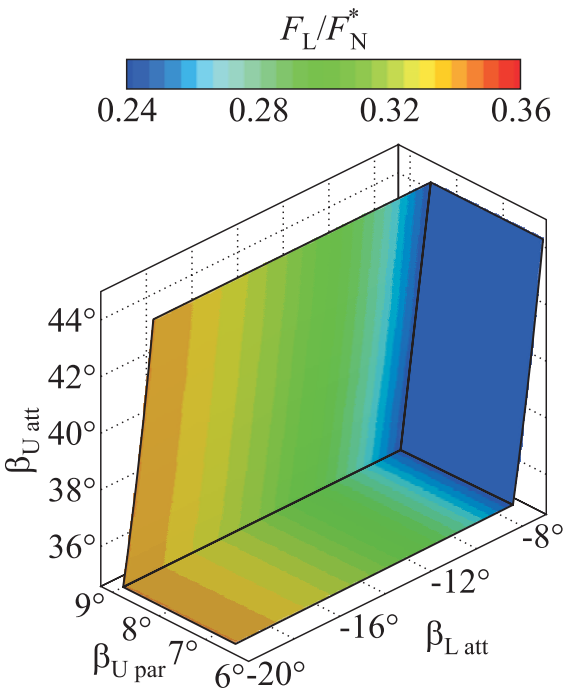

(a)

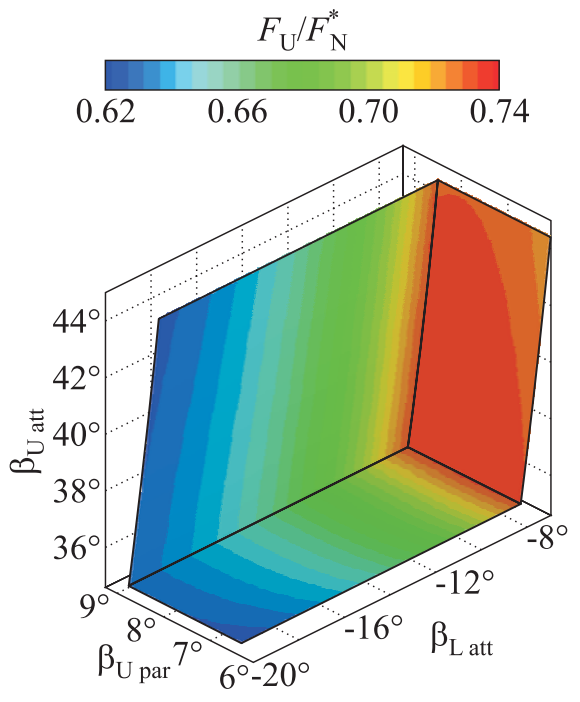

(b)

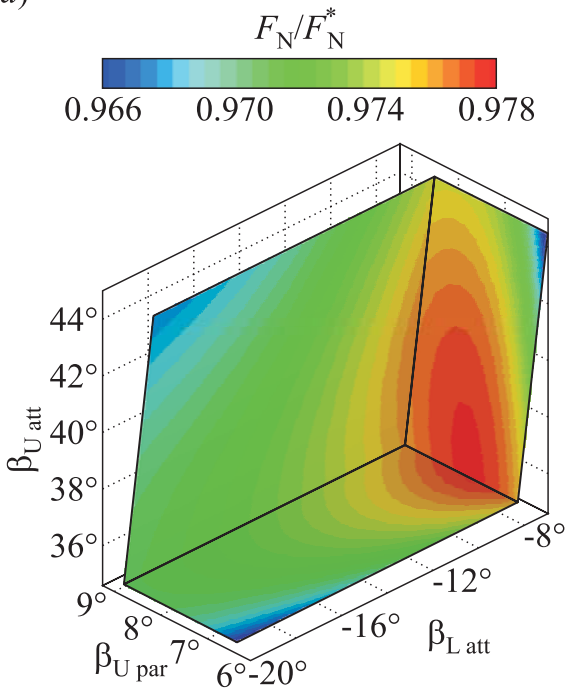

(c)

Figure 4 Normalized pressure forces for $D C B=0.4 \mathrm{~m}$ and cubic upper contour: lower contour $(a)$; upper contour $(b)$; and their sum $(c)$. (Refer Davidenko et al., p. 685.) 

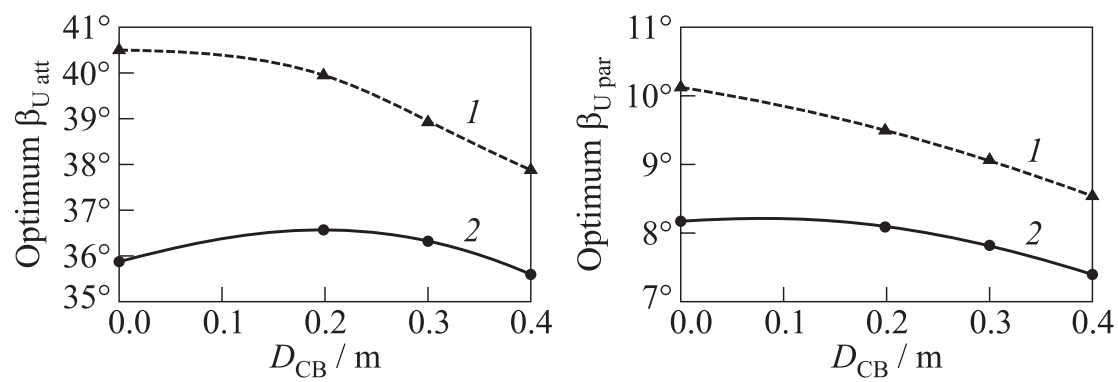

(a)

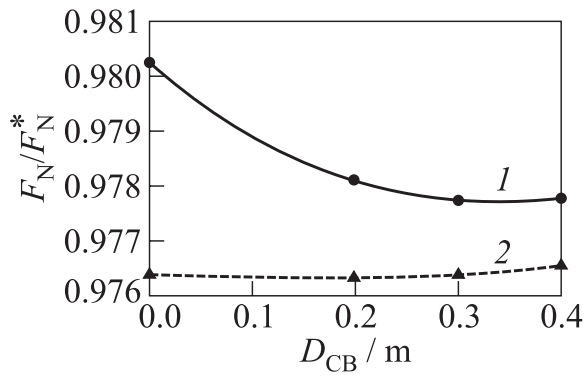

(b)

Figure 5 Optimum contour angles $(a)$ and normalized nozzle thrust $(b)$ vs. center body diameter for quadratic (1) and cubic (2) upper contours

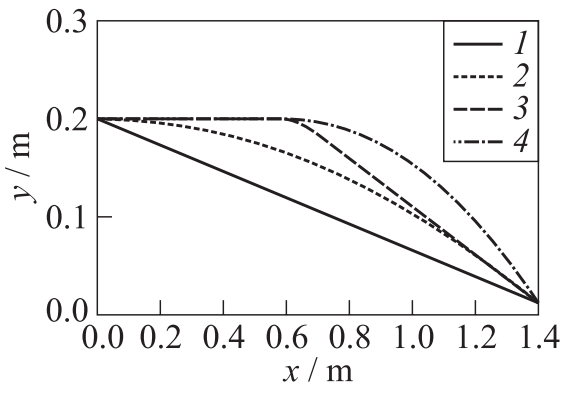

Figure 6 Various ogive shapes of the center body for $D_{\mathrm{CB}}=0.4 \mathrm{~m}: 1-L_{\mathrm{cyl}}=0 \mathrm{~m}$, $R_{\mathrm{L}}=0.13 \mathrm{~m} ; 2-L_{\mathrm{cyl}}=0 \mathrm{~m}, R_{\mathrm{L}}$ $=5.25 \mathrm{~m} ; 3-L_{\mathrm{cyl}}=0.6 \mathrm{~m}, R_{\mathrm{L}}=0.3 \mathrm{~m}$; and $4-L_{\mathrm{cyl}}=0.6 \mathrm{~m}, R_{\mathrm{L}}=1.78 \mathrm{~m}$
Optimum nozzle shapes have been designed for different diameters of the center body. The best shape of the center body contour was always straight with the maximum allowed length, $x_{\mathrm{L} \text { par }}=1.4 \mathrm{~m}$. In Fig. 5, the optimum values of $\beta_{U \text { att }}$ and $\beta_{U \text { par }}$ and corresponding normalized thrust, $F_{\mathrm{N}} / F_{\mathrm{N}}^{*}$, are plotted vs. $D_{\mathrm{CB}}$ for quadratic and cubic upper contours. One can note that the cubic contour has always lesser angles $\beta_{\mathrm{U} \text { att }}$ and $\beta_{\mathrm{U} \text { par }}$ than the quadratic one. Within the range $D_{\mathrm{CB}}=0.2-0.4 \mathrm{~m}$, optimum angles demonstrate decreasing trends for both contour kinds. Thrust obtained with a center body is slightly 
lower with respect to the classical nozzle $\left(D_{\mathrm{CB}}=0\right)$ in the case of cubic contour and independent of $D_{\mathrm{CB}}$ in the case of quadratic contour. The overall thrust difference is $0.15 \%-0.4 \%$.

According to the results presented in Fig. 4, the maximum thrust corresponds to the limiting case for the center body having a straight contour. This suggests an idea to try ogive shapes, for example, a fully circular contour or contours with a cylindrical portion, as shown in Fig. 6 for $D_{\mathrm{CB}}=0.4 \mathrm{~m}$. It should be noted that the optimum upper contour is sensitive to the center body shape up to $x_{\mathrm{L}}$ $\approx 0.6 \mathrm{~m}$. Beyond this length, the center body shape is important only for the force applied to the lower contour, $F_{\mathrm{L}}$.

Nozzle optimization has been performed for center bodies with a cylindrical portion and two shapes of the converging portion: straight with relatively small $R_{\mathrm{L}}$ and circular with maximum possible $R_{\mathrm{L}}$. The results obtained for variable $L_{\text {cyl }}$ and different $D_{\mathrm{CB}}$ are shown in Fig. 7. The optimum values of $\beta_{\mathrm{U} \text { att }}$ are virtually equal to $38^{\circ}$ for all cases except for the straight contour with $L_{\text {cyl }}=0$ and $0.1 \mathrm{~m}$. One can see in Fig. $7 b$ that the fraction represented by $F_{L}$ in the nozzle thrust drastically reduces when $L_{\text {cyl }}$ increases. This is because the converging portion of the center body shifts toward the low-pressure zone. Nevertheless, the nozzle thrust increases due to the rise of $F_{\mathrm{U}}$. Depending on $D_{\mathrm{CB}}$, maximum thrust is obtained at $L_{\text {cyl }}=0.4-0.5 \mathrm{~m}$. The circular shape is better at lesser $L_{\text {cyl }}$ whereas the

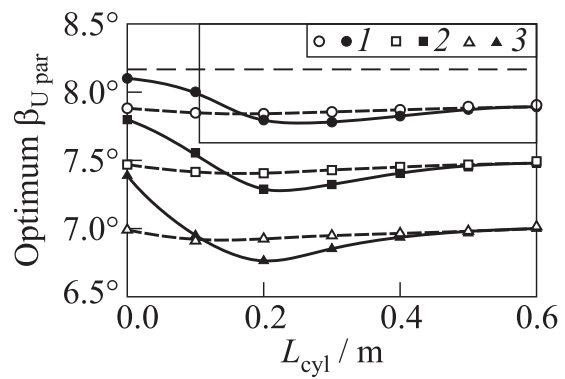

(a)

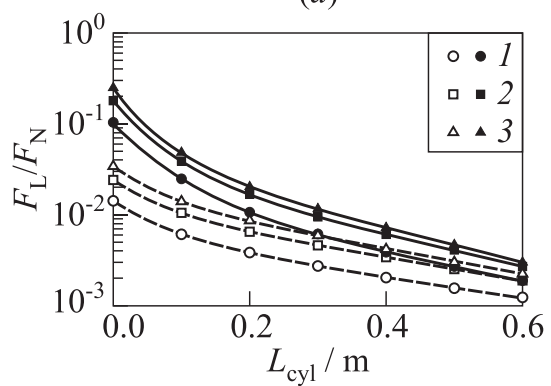

(b)

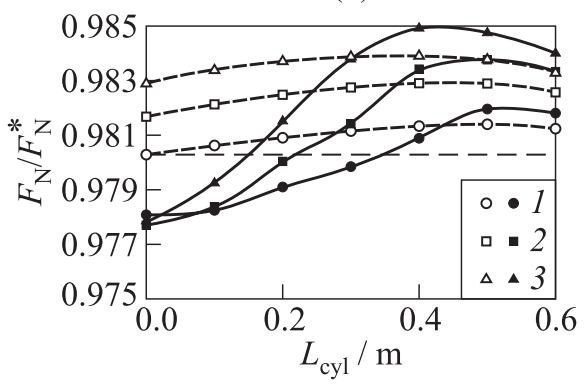

(c)

Figure 7 Optimum contour angle at the end point $(a), F_{L}$ fraction in the nozzle thrust $(b)$, and normalized nozzle thrust $(c)$ vs. length of the center-body cylindrical portion. Horizontal lines mark the level corresponding to the classical nozzle. Black signs refer to straight and empty signs to circular lower contours: $1-D_{\mathrm{CB}}=0.2 \mathrm{~m} ; 2-0.3 ;$ and $3-$ $D_{\mathrm{CB}}=0.4 \mathrm{~m}$ 
straight shape has an advantage at greater $L_{\text {cyl }}$. One can also note that with an ogive center body, the maximum thrust exceeds the level corresponding to the classical nozzle (Fig. $7 c$ ).

\subsection{Nozzle Thrust with Viscous Losses}

From the previous analysis of thrust losses, one can see that viscous effects represent a very important factor that must be taken into account when choosing the best nozzle contour. This subsection provides an analysis based on the results of NS simulations performed for several optimized contours.

The nozzles with cubic upper contours and straight lower contours, for which optimization results were presented in Fig. 5, will be considered first. For these nozzles, Euler and NS results on $F_{p \mathrm{~N}} / F_{\mathrm{N}}^{*}, F_{\nu \mathrm{N}} / F_{\mathrm{N}}^{*}$, and $F_{\mathrm{N}} / F_{\mathrm{N}}^{*}$ as functions of $D_{\mathrm{CB}}$ are shown in Fig. 8. Forces $F_{p \mathrm{~N}}$ and $F_{\nu \mathrm{N}}$ represent, respectively, the total pressure and viscous forces integrated along the lower and upper contours. The nozzle thrust was defined as $F_{\mathrm{N}}=F_{p \mathrm{~N}}+F_{\nu \mathrm{N}}$. The NS results are given for the cases of adiabatic and cooled walls. With respect to the Euler simulations, $F_{p \mathrm{~N}}$ is $1 \%$ to $4 \%$ higher because the flow expansion in the nozzle is reduced by the growing boundary layers. Accounting for $F_{\nu \mathrm{N}}$ that represents $-5 \%$ to $-8 \%$, net losses in $F_{\mathrm{N}}$ are $2.6 \%-3 \%$ for adiabatic walls and $4.6 \%-6.2 \%$ for cooled walls (higher losses correspond to larger $D_{\mathrm{CB}}$ ).

The NS results, corresponding to ogive shapes of the center body (see Figs. 6 and 7 ) are shown in Fig. 9. Here, $F_{\nu \mathrm{L}} / F_{\nu \mathrm{N}}, F_{\nu \mathrm{N}} / F_{\mathrm{N}}^{*}$, and $F_{\mathrm{N}} / F_{\mathrm{N}}^{*}$ are plotted vs. $L_{\text {cyl }}$ for different diameters $D_{\mathrm{CB}}=0.2-0.4 \mathrm{~m}$ with $F_{\nu \mathrm{L}}$ standing for the viscous force applied to the lower contour. One can note that the center body creates a drag, which represents an almost constant fraction of $F_{\nu \mathrm{N}}$ when $L_{\text {cyl }}$ increases. The net force $F_{\mathrm{L}}=F_{p \mathrm{~L}}+F_{\nu \mathrm{L}}$ is negative at $L_{\text {cyl }} \geq 0.3 \mathrm{~m}$. Ogive shapes with circular converging portion provide much more considerable drag at $L_{\text {cyl }}=0$. As a result, these shapes have no advantage at any $L_{\text {cyl }}$. Ogive shapes with straight converging portion can give a little better performance at $D_{\mathrm{CB}}=0.2 \mathrm{~m}$ and are definitely disadvantageous at $D_{\mathrm{CB}}=0.4 \mathrm{~m}$. Taking into account the weight and wall cooling, the case of straight contour with $L_{\mathrm{cyl}}=0$ must be finally preferred.

\subsection{Nozzle Flowfield}

To illustrate the nozzle flowfield, Mach number fields are shown in Fig. 10 for two center bodies with straight contours: $D_{\mathrm{CB}}=0.2$ and $0.4 \mathrm{~m}$. The flowfields above and below the axis respectively correspond to the Euler and NS simulations.

The flow is expanding everywhere except for the zone near the axis. Because of the flow convergence near the center body, a conical shock front forms downstream from its tip. This shock is clearly marked by iso-Mach lines inflection in 


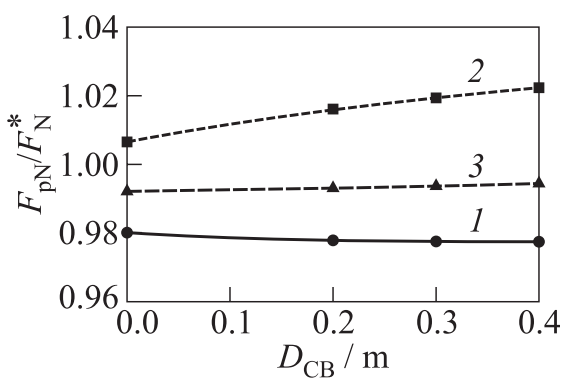

(a)

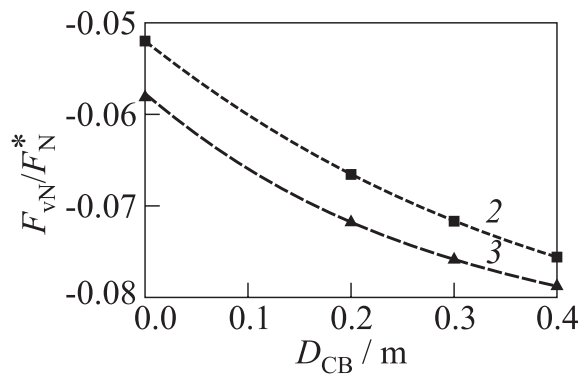

(b)

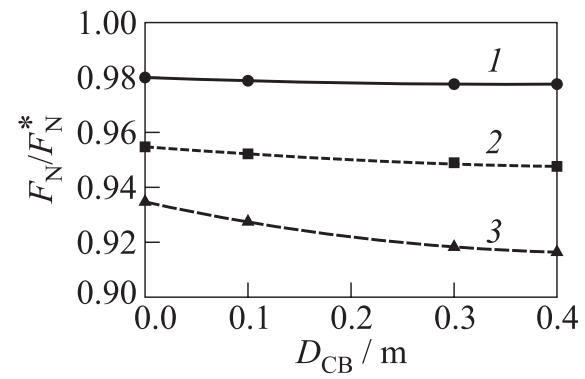

(c)

Figure 8 Normalized total pressure force $(a)$, total viscous force $(b)$, and nozzle thrust $(c)$ vs. diameter of the center body from Euler (1) and NS simulations: 2 - adiabatic and $3-T_{w}=1300 \mathrm{~K}$

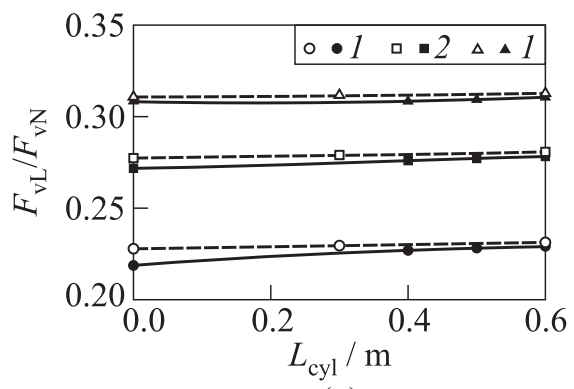

(a)

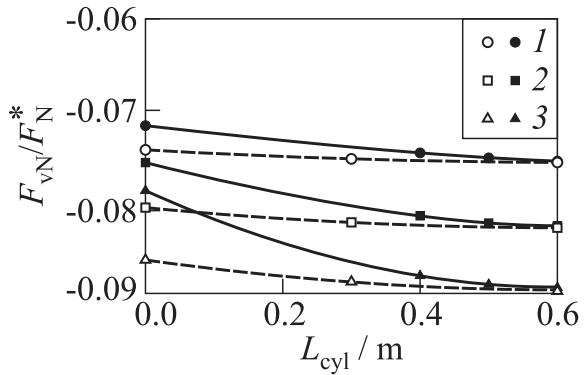

(b)

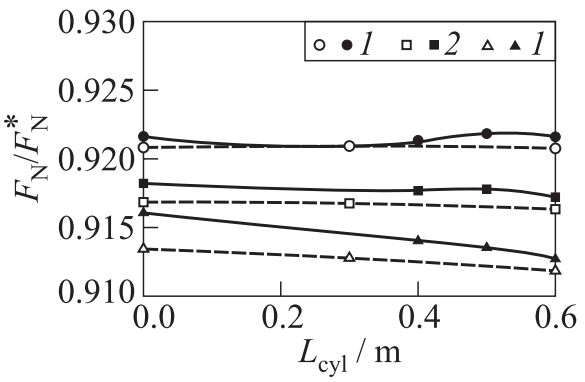

(c)

Figure 9 Fraction of the center body viscous force in the total viscous force $(a)$, normalized total viscous force $(b)$, and normalized nozzle thrust $(c)$ vs. length of the center-body cylindrical portion. Black signs refer to straight and empty signs to circular lower contours: 1 $D_{\mathrm{CB}}=0.2 \mathrm{~m} ; 2-0.3$; and $3-D_{\mathrm{CB}}$ $=0.4 \mathrm{~m}$ 


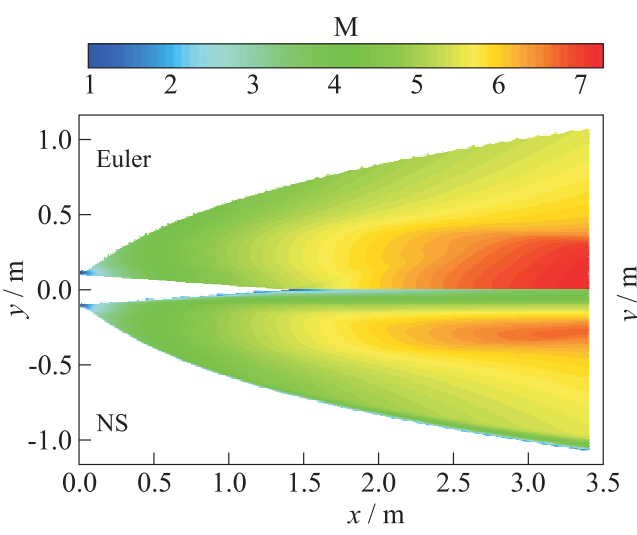

(a)

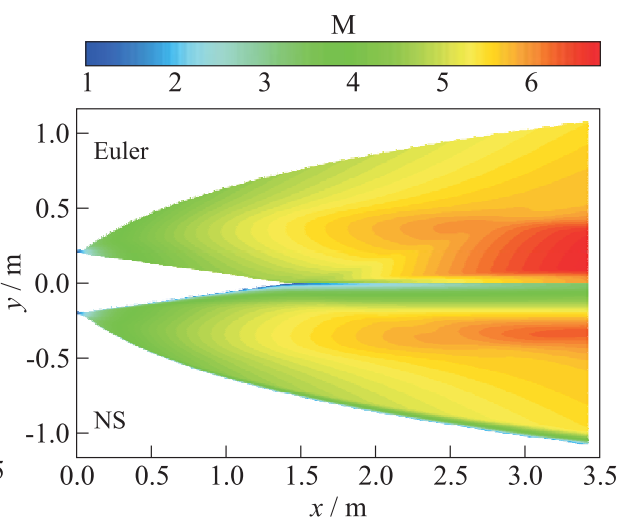

(b)

Figure 10 Mach number fields for $D_{\mathrm{CB}}=0.2 \mathrm{~m}(a)$ and $0.4 \mathrm{~m}(b)$ from Euler (above axis) and NS (below axis) simulations. (Refer Davidenko et al., p. 690.)

the fields obtained from the Euler simulations. For the NS results, the boundary layers on the nozzle walls are clearly seen. The displacement effect, which reduces flow expansion, is manifested by lower Mach numbers toward the nozzle exit. The boundary layer grows quicker on the center body due to the flow convergence. Because of a thick boundary layer, the flow deviation is smoother and the conical shock is weaker. The flow compression is not strong enough to provoke a separation. Past the center body tip, the boundary layer transforms to a free wake.

\section{CONCLUDING REMARKS}

A numerical study has been conducted to determine optimum shapes of axisymmetric nozzles with a center body. Several optimized contours have been designed based on Euler simulations for different diameters and shapes of the center body. The highest thrust is obtained for an ogive shape consisting of a cylindrical part and a straight converging part.

For the optimized contours, NS simulations have been performed in order to take into account viscous losses due to skin friction and heat exchange. According to the obtained results, the viscous effects are responsible for a 3 to 6 percent thrust reduction with respect to the Euler results. Among the considered configurations of the center body, the case of straight contour without cylindrical part is preferable. 


\section{ACKNOWLEDGMENTS}

The financial support of MBDA-France, the Centre National de la Recherche Scientifique (CNRS), the FEDER and FSE funds of the European Commission, the Region Centre, the Departement of Cher, the Communauté d'Agglomeration Bourges Plus, and the Prefecture of Region Centre is greatly acknowledged.

\section{REFERENCES}

1. Bykovskii, F. A., S. A. Zhdan, and E. F. Vedernikov. 2006. Continuous spin detonations. J. Propul. Power 22:1204-16.

2. Vuillermoz, P., C. Weiland, G. Hagemann, B. Aupoix, et al. 2004. Nozzle desing and optimization. In: Liquid rocket thrust chambers: Aspects of modeling, analysis, and design. Eds. V. Yang, M. Habiballah, J. Hulka, and M. Popp. Progress in aeronautics and astronautics ser. 200:469-92.

3. Rao, G. V.R. 1958. Exhaust nozzle contour for optimum thrust. Jet Propul. 28(6):377-82.

4. Rao, G. V.R. 1961. Spike nozzle contour for optimum thrust. Planetary Space Sci. 4:92-101.

5. Hoffman, J. D., M. P. Scofield, and H. D. Thompson. 1972. Thrust nozzle optimization including boundary layer effects. J. Optimization Theory Appl. 10(3):133-59.

6. Hoffman, J. D. 1967. A general method for determining optimum thrust nozzle contours for chemical reacting gas flows. AIAA J. 5(4):670-76.

7. Allman, J. G., and J. D. Hoffman. 1981. Design of maximum thrust nozzle contours by direct optimisation methods. AIAA J. 19(6):750-51.

8. Wang, C.-H., Y. Liu, and L.-Z. Qin. 2009. Aerospike nozzle contour design and its performance validation. Acta Astronautica 64(11-12):1264-75.

9. Takovitskii, S.A. 2009. Optimal supersonic nozzles with power-law generators. Fluid Dyn. 44(1):129-34.

10. Keeling, S.L. 1993. A strategy for the optimal design of nozzle contours. AIAA Paper No. 93-2720.

11. Cai, G., J. Fang, X. Xu, and M. Liu. 2007. Performance prediction and optimization for liquid rocket engine nozzle. Aerospace Sci. Technol. 11(2-3):155-62.

12. Press, W.H., S. A. Teukolsky, W. T. Vetterling, and B. P. Flannery 1992. Numerical recipes in FORTRAN: The art of scientific computing. 2nd ed. Cambridge University Press.

13. Anderson, D., J. Tannehill, and R. Pletcher. 1984. Computational fluid mechanics and heat transfer. New York: Hemisphere.

14. Davidenko, D. M., I. Gökalp, E. Dufour, and P. Magre 2006. Systematic numerical study of the supersonic combustion in an experimental combustion chamber. AIAA Paper No. 2006-7913. 
15. Burcat, A., and B. Ruscic. 2005. Third millenium ideal gas and condensed phase thermochemical database for combustion with updates from active thermochemical tables. Argonne National Laboratory Report No. ANL-05/20; Technion Report No. TAE 960.

16. Bird, R. B., W.E. Stewart, and E. N. Lightfoot. 1960. Transport phenomena. New York: John Wiley and Sons.

17. Wilcox, D. C. 1998. Turbulence modeling for CFD. DCW Industries, Inc.

18. Vinci Rocket Engine — Thrust Chamber. http://cs.astrium.eads.net/sp/ LauncherPropulsion/Vinci-Rocket-Engine.html.

19. Zucrow, M. J., and J. D. Hoffman. 1977. Gas dynamics. New York: John Wiley and Sons. II. 